Research Article

\title{
The Predictive Value of Serum Uric Acid on Acute Kidney Injury following Traumatic Brain Injury
}

\author{
Ruo Ran Wang, Min He, Xiao Feng Ou, Xiao Qi Xie, and Yan Kang $(\mathbb{D}$ \\ Department of Critical Care Medicine, West China Hospital, Sichuan University, Chengdu, 610041 Sichuan Province, China \\ Correspondence should be addressed to Yan Kang; kangyan@scu.edu.cn
}

Received 26 February 2020; Revised 14 July 2020; Accepted 5 August 2020; Published 31 August 2020

Academic Editor: Dirk Bandorski

Copyright ( 2020 Ruo Ran Wang et al. This is an open access article distributed under the Creative Commons Attribution License, which permits unrestricted use, distribution, and reproduction in any medium, provided the original work is properly cited.

\begin{abstract}
Backgrounds. Acute kidney injury (AKI) is a prevalent nonneurological complication in patients with traumatic brain injury (TBI). We designed this study to explore the association between serum uric acid (SUA) level and the occurrence of AKI following TBI. Methods. This is a retrospective single-center study. A total of 479 patients admitted with TBI were included in this study. We utilized SUA and other risk factors for AKI to construct a predictive model by performing multivariate logistic regression. 374 patients and 105 patients were, respectively, divided into a training set and validation set. The predictive value of the single SUA and constructed model was evaluated by drawing a receiver operating characteristic (ROC) curve. AKI was diagnosed according to the KIDGO criteria. Results. 79 (21.12\%) patients were diagnosed with AKI in the training cohort. The patients in the AKI group are older than those in the non-AKI group $(p=0.01)$. And the Glasgow Coma Scale (GCS) of the AKI group was lower than that of the non-AKI group $(p<0.001)$. In a multivariate logistic regression analysis, we found that heart rate $(p=0.041)$, shock $(p=0.018)$, serum creatinine $(p<0.001)$, and serum uric acid (SUA) $(p<0.001)$ were significant risk factors for AKI. Bivariate correlation analyses showed that serum creatinine was moderately positively correlated with SUA $(r=0.523, p<0.001)$. Finally, the area under the receiver operating characteristic curve (AUC) of SUA for predicting AKI in the training set and validation set was 0.850 (0.805-0.895) and 0.869 (0.801-0.938), respectively. Conclusions. SUA is an effective risk factor for AKI following TBI. Combining SUA with serum creatinine could more accurately identify TBI patients with high risk of developing AKI.
\end{abstract}

\section{Introduction}

It has been estimated that 10 million people would suffer a traumatic brain injury (TBI) event every year all over the world [1]. More than 1.4 million people may undergo TBI annually in the USA, and 50,000 of them will die due to this kind of severe injury [2]. In addition to the neurological damage of TBI, nonneurological complications could also contribute to the mortality and unfavored outcome [3]. Acute kidney injury, a form of nonneurological complications, has been paid much attention in a neurointensive care unit (NICU). Both pathophysiological effects of secondary brain injury and side effects of therapy could aggravate the development of AKI after TBI [4]. Previous studies have shown that the incidence of AKI following TBI ranged from $3.9 \%$ to $24 \%$ [5-8]. And the association between occurrence of AKI and increased mortality and prolonged length of hos- pital stay (LOS) has been verified $[5,9,10]$. To avoid the adverse effect of AKI on the outcome of TBI patients, predicting the development of AKI in an early stage is necessary for neurosurgeons and intensivists to reduce the use of drugs with high nephrotoxicity and convert patients to drugs which have less adverse effects on renal function. For reducing intracranial pressure, more glycerol fructose and hypertonic saline should be used rather than mannitol with relatively serious side effects on the kidney.

Uric acid, an end product of purine metabolism, has been confirmed to be neuroprotective because of its antioxidant property. Previous studies have shown that elevated serum uric acid (SUA) level was associated with a favorable outcome in patients with ischemic stroke, intracerebral hemorrhage, or TBI $[11,12]$. However, the correlation between AKI, poor progression of chronic kidney disease (CKD), and hyperuricemia has been verified. Uric acid could impair 
renal tubular endothelial function and cause subtle renal damage by intratubular crystal precipitation as well as activation of oxidative stress and renal inflammation [13, 14]. There are several studies examining the association between SUA and the occurrence of AKI in various patients including those undergoing radical cystectomy and those undergoing cardiac surgery [15-17]. However, the predictive value of SUA on AKI following TBI has not been examined. We performed this study to determine whether SUA was valuable in predicting AKI in patients with TBI and explored the correlation between SUA and other factors.

\section{Materials and Methods}

2.1. Patients. This is a single-center cohort study. We included patients diagnosed with TBI and hospitalized in West China Hospital within 24 hours after injuries between January 2015 and October 2019. The diagnosis of TBI was established by analyzing medical history and clinical manifestation and was confirmed by reviewing images including computed tomography (CT) and magnetic resonance imaging (MRI). Neurosurgeons and intensivists provided patients with reasonable treatments according to the current guidelines. Patients simultaneously diagnosed with other central nervous system diseases, hematological system diseases, cardiovascular diseases, tumor diseases, metabolic diseases, chronic hepatorenal diseases, and pregnancy were excluded. Patients who lack laboratory and clinical data on the first day and those lost to follow-up were also excluded from this study. Finally, 479 patients were included in this retrospective observational study. To explore and verify the predictive value of risk factors and predictive models, 374 patients admitted between January 2015 and September 2018 were selected as a training cohort and 105 patients admitted between October 2018 and October 2019 were selected as a validation cohort. The study was approved by the ethics committee of West China Hospital. All patients provided written informed consent.

2.2. Data Collection. Records of prehospital time were collected by emergency workers. Vital signs and the Glasgow Coma Scale (GCS) in admission were collected through inquiring the electronic medical record (EMR) system. A shock state was evaluated and recorded by nurses on the first day of admission. SUA level and other laboratory indexes were obtained by analyzing blood specimens on the first day of admission. AKI was evaluated after 48 hours after admission according to the KIDGO criteria [18].

2.3. Statistical Analysis. Normally distributed data was presented as mean \pm standard deviation while nonnormally distributed data was presented as median (interquartile range). The Kolmogorov-Smirnov test was used to confirm the normality of the included variables. Categorical data was presented as numbers (percentage). We used independent Student's $t$-test to compare the difference between two groups of normally distributed variables. And the MannWhitney $U$ test was used to compare the difference between two groups of nonnormally distributed variables. The differ- ence of categorical variables was analyzed by using the $\chi^{2}$ test. Multivariate logistic regression analysis was utilized to analyze the association between various factors and the occurrence of AKI. The odds ratio (OR) and 95\% confidence intervals (CI) of each risk factor were also calculated. We performed Spearman's method to analyze the correlation of serum uric acid level and other laboratory variables. To testify the value of different models for predicting AKI, we have drawn the receiver operating characteristic (ROC) curve and calculated the area under the curve (AUC), sensitivity, and specificity. Finally, the $Z$ test was utilized to test the difference of AUC.

A $p$ value $<0.05$ was considered to be of statistical significance. SPSS 22.0 Windows software (SPSS, Inc., Chicago, IL) was used for all statistical analyses.

\section{Results}

3.1. Baseline Characteristics of AKI Patients and Non-AKI Patients. As shown in Table 1, among 374 patients diagnosed with TBI in the training cohort, 79 (21.12\%) patients developed AKI. The age of the AKI group was 47 (32-60) while that of the non-AKI group was $41(23-54)(p=0.01)$. The female percentage did not differ between the non-AKI group and the AKI group $(p=0.433)$. The prehospital time of the AKI group was shorter than that of the non-AKI group $(p=0.028)$. Comparing vital signs in admission, we found that the heart rate of the AKI group was higher $(p=0.015)$ and the respiratory rate of the AKI group was lower $(p=0.022)$ than those of the non-AKI group. The GCS score of all patients was 6 (5-9), indicating that the included patients were mostly diagnosed with moderate and severe TBI. The AKI group had significantly lower GCS than the non-AKI group $(p<0.001)$. In addition, patients in the AKI group were more likely to develop a shock state than patients in the non-AKI group $(p<0.001)$. The results of the laboratory tests showed that only white blood cell (WBC) count was not significantly different between the two groups. The AKI group has higher level of glucose $(p<0.001)$, chlorine $(p<0.001)$, total bilirubin $(p=0.001)$, serum urea $(p<0.001)$, serum creatinine $(p<0.001)$, and SUA $(p<0.001)$. Instead, the non-AKI group has higher level of platelet $(p<0.001)$, hemoglobin $(p<0.001)$, albumin $(p<0.001)$, and cholesterol $(p<0.001)$. In consideration of several drugs for reducing intracranial pressure (ICP), only the utilization rate of hypertonic saline was different between these two groups $(p=0.004)$. Patients in the AKI group were less likely to receive hypertonic saline. Finally, patients who developed AKI could suffer increased in-hospital mortality $(p<0.001)$ and unfavorable 90-day GOS $(p<0.001)$. The median length of ICU stay in overall patients was 2 days. The length of ICU stay did not significantly differ between the non-AKI group and the AKI group $(p=0.384)$. The median length of hospital stay was 11 days. Compared with the non-AKI group, the AKI group had shorter length of hospital stay $(p=0.001)$.

3.2. Multivariate Logistic Regression Analysis of Risk Factors for AKI. Statistically significant variables in baseline comparison were selected for subsequent multivariate logistic 
TABLE 1: Baseline characteristics of AKI patients and non-AKI patients.

\begin{tabular}{|c|c|c|c|c|}
\hline & Total $(n=374)$ & Non-AKI $(n=295)$ & AKI $(n=79)$ & $p$ \\
\hline Age (year) & $43(25-57)$ & $41(23-54)$ & $47(32-60)$ & 0.010 \\
\hline Female $(n, \%)$ & $93(24.87)$ & $76(25.76 \%)$ & $17(21.52 \%)$ & 0.433 \\
\hline Prehospital time (hour) & $1(1-2)$ & $1(1-2)$ & $1(1-1)$ & 0.028 \\
\hline \multicolumn{5}{|l|}{ Vital signs in admission } \\
\hline SBP (mmHg) & $120(105-138)$ & $120(106-138)$ & $113(102-133)$ & 0.135 \\
\hline $\mathrm{DBP}(\mathrm{mmHg})$ & $71.51 \pm 17.103$ & $72.34 \pm 16.293$ & $68.41 \pm 19.641$ & 0.104 \\
\hline Heart rate (bpm) & $100(81-119.25)$ & $98(80-117)$ & $107(89-124)$ & 0.015 \\
\hline Temperature $\left({ }^{\circ} \mathrm{C}\right)$ & $36.7(36.5-37.1)$ & $36.7(36.5-37)$ & $36.8(36.4-37.5)$ & 0.368 \\
\hline Respiratory rate & $20(16-22)$ & $20(16-23)$ & $18(15-22)$ & 0.022 \\
\hline GCS in admission & $6(5-9)$ & $7(5-10)$ & $5(4-6)$ & $<0.001$ \\
\hline Shock (\%) & $116(31.02 \%)$ & $71(24.07 \%)$ & $45(56.96 \%)$ & $<0.001$ \\
\hline \multicolumn{5}{|l|}{ Laboratory tests } \\
\hline $\operatorname{WBC}\left(10^{9} / \mathrm{L}\right)$ & $14.34(10.33-19)$ & $13.98(10.35-18.92)$ & $14.98(10.15-20.17)$ & 0.607 \\
\hline Platelet $\left(10^{9} / \mathrm{L}\right)$ & $106.5(68-171.25)$ & $122(76-180)$ & $72(44-111)$ & $<0.001$ \\
\hline Hemoglobin (g/L) & $90(76-110.25)$ & $94(79-112)$ & $80(71-96)$ & $<0.001$ \\
\hline Albumin (g/L) & $31.60 \pm 8.008$ & $32.60 \pm 7.867$ & $27.86 \pm 7.445$ & $<0.001$ \\
\hline Glucose (mmol/L) & $9.49(7.04-13.14)$ & $8.84(6.68-12.53)$ & $11.7(8.9-16.77)$ & $<0.001$ \\
\hline Chlorine $(\mathrm{mmol} / \mathrm{L})$ & $110.9(105.58-118.93)$ & $109.3(104.3-116.6)$ & $116.9(111.8-128.9)$ & $<0.001$ \\
\hline Cholesterol (mmol/L) & $2.75(1.97-3.62)$ & $2.89(2.22-3.68)$ & $1.97(1.35-2.79)$ & $<0.001$ \\
\hline Total bilirubin $(\mu \mathrm{mol} / \mathrm{L})$ & $14.6(10.1-20.35)$ & $13.9(9.4-19.65)$ & $17.5(12.1-22.7)$ & 0.001 \\
\hline Serum urea $(\mathrm{mmol} / \mathrm{L})$ & $6.36(4.93-8.64)$ & $5.93(4.65-7.77)$ & $9.4(7.11-15.3)$ & $<0.001$ \\
\hline Serum creatinine $(\mu \mathrm{mol} / \mathrm{L})$ & $71(51-96.25)$ & $65(48-82)$ & $133(97-237)$ & $<0.001$ \\
\hline Uric acid $(\mu \mathrm{mol} / \mathrm{L})$ & $288(186.75-388.5)$ & $249(165-347)$ & $452.5(360-585)$ & $<0.001$ \\
\hline \multicolumn{5}{|l|}{ Drugs for reducing ICP } \\
\hline Hypertonic saline (\%) & $91(24.33 \%)$ & $81(27.46 \%)$ & $10(12.66 \%)$ & 0.004 \\
\hline Mannitol (\%) & $257(68.72 \%)$ & $203(68.81 \%)$ & $54(68.35 \%)$ & 0.938 \\
\hline Glycerol fructose (\%) & $30(8.02 \%)$ & $23(7.80 \%)$ & $7(8.86 \%)$ & 0.760 \\
\hline Furosemide (\%) & $55(14.71 \%)$ & $39(13.22 \%)$ & $16(20.25 \%)$ & 0.129 \\
\hline In-hospital mortality (\%) & $187(50 \%)$ & $119(40.34 \%)$ & $68(80.08 \%)$ & $<0.001$ \\
\hline 90-day GOS & $2(1-3)$ & $3(1-4)$ & $1(1-1)$ & $<0.001$ \\
\hline Length of ICU stay (day) & $2(1-16)$ & $2(0-17)$ & $2(1-11)$ & 0.384 \\
\hline Length of hospital stay (day) & $11(4-27)$ & $13(5-28)$ & $6(3-14)$ & 0.001 \\
\hline
\end{tabular}

regression analysis. As shown in Table 2, four factors including heart rate (OR 0.984, 95\% CI [0.969-0.999]), shock (OR 2.905, 95\% CI [1.201-7.028]), serum creatinine (OR 1.033\% CI [1.017-1.048]), and SUA (OR 1.008, 95\% CI [1.0041.011]) were statistically significant.

\subsection{Bivariate Correlation Analyses of Uric Acid and Various} Clinical and Laboratory Parameters. Spearman's method was performed to analyze the correlation between other parameters and SUA. We found that heart rate $(r=0.163$, $p=0.002)$, shock $(r=0.204, p<0.001)$, WBC $(r=0.237$, $p<0.001)$, glucose $(r=0.285, p<0.001)$, chlorine $(r=0.286$, $p<0.001)$, and serum urea $(r=0.291, p<0.001)$ were weakly positively correlated with SUA (Table 3 ). And GCS $(r=-0.204, p<0.001)$, platelet $(r=-0.264, p<0.001)$, albumin $(r=-0.126, p=0.015)$, and cholesterol $(r=-0.184$, $p<0.001)$ were weakly negatively correlated with SUA.
Furthermore, serum creatinine was moderately positively associated with SUA $(r=0.523, p<0.001)$.

3.4. Comparison of the Predictive Value of Different Factors and Models to Predict AKI in the Training Cohort and Validation Cohort. In the training cohort, the AUC of SUA and serum creatinine was 0.850 and 0.881 , respectively (Table 4) (Figure 1). There was no difference in AUC between SUA and serum creatinine $(Z=0.9126, p>0.05)$. The AUC of combining SUA with serum creatinine was 0.917 , which was higher than that of SUA alone $(Z=2.246$, $p<0.05)$. Then, we use heart rate, shock, serum creatinine, and SUA which were statistically significant in multivariate logistic analysis, to construct predictive model 1. The AUC of model 1 was 0.935 , which was not significantly higher than the AUC of combining SUA with serum creatinine $(Z=0.7247, p>0.05)$. Finally, the AUC of GCS was 0.686, 
TABLE 2: Multivariate logistic regression analysis of factors associated with AKI.

\begin{tabular}{lccc}
\hline & OR & $95 \%$ CI & $p$ \\
\hline Age & 1.015 & $0.989-1.041$ & 0.261 \\
Prehospital time & 0.730 & $0.482-1.104$ & 0.136 \\
Heart rate & 0.984 & $0.969-0.999$ & 0.041 \\
Respiratory rate & 0.982 & $0.911-1.060$ & 0.646 \\
GCS in admission & 1.050 & $0.935-1.179$ & 0.412 \\
Shock & 2.905 & $1.201-7.028$ & 0.018 \\
Platelet & 1.000 & $0.991-1.009$ & 0.963 \\
Hemoglobin & 0.995 & $0.975-1.015$ & 0.612 \\
Albumin & 1.005 & $0.935-1.081$ & 0.882 \\
Glucose & 1.078 & $0.993-1.170$ & 0.073 \\
Chlorine & 1.018 & $0.982-1.055$ & 0.341 \\
Cholesterol & 0.827 & $0.502-1.364$ & 0.457 \\
Total bilirubin & 1.031 & $0.993-1.071$ & 0.115 \\
Serum urea & 1.148 & $0.995-1.323$ & 0.058 \\
Serum creatinine & 1.033 & $1.017-1.048$ & $<0.001$ \\
Uric acid & 1.008 & $1.004-1.011$ & $<0.001$ \\
Hypertonic saline & 0.789 & $0.247-2.525$ & 0.690 \\
\hline
\end{tabular}

OR: odds ratio; CI: confidence interval; GCS: Glasgow Coma Scale.

which was significantly lower than that of SUA $(Z=4.338$, $p<0.05)$. In the validation cohort, the AUC of SUA and serum creatinine was 0.869 and 0.868 , respectively (Table 4 ) (Figure 2). And the AUC of combining uric acid with creatinine was 0.905 , which was higher than 0.900 of constructed model 1 and 0.869 of uric acid though without statistical significance $(Z=0.0996, p>0.05 ; Z=0.7273, p>0.05)$. The AUC of GCS for predicting AKI was 0.610 in the validation cohort.

\section{Discussion}

The aim of this study was to confirm the predictive value of SUA in AKI following TBI. By performing multivariate logistic regression analysis, we found that heart rate in admission, shock, serum creatinine, and SUA were risk factors for the development of AKI. ROC curves showed that SUA had a comparable predictive value with serum creatinine alone. In our study, the percentage of patients developing AKI was $21.12 \%$ according to the KIDGO criteria. Previous studies reported that the incidence of AKI following TBI ranged from $0.45 \%$ to $35 \%[3,19-21]$. This variability might originate from different diagnostic criteria for AKI, severity of the included patients, and different medical level. We found that patients who developed AKI had higher age and lower GCS, which was consistent with the results of previous studies $[6,7]$. Baseline comparison indicated that the occurrence rate of shock was higher in the AKI group. This was coincident with the opinion that developing AKI was usually aggravated by reduced renal perfusion pressure secondary to shock or hypovolemia [22]. In addition, the fact that the heart rate of the AKI group was higher than that of the non-AKI group could be explained by the complementary response to hypo-
TABLE 3: Bivariate correlation analyses of uric acid and various clinical and laboratory parameters.

\begin{tabular}{lcc}
\hline Variables & $r$ & $p$ \\
\hline Age & 0.088 & 0.090 \\
Male & 0.029 & 0.573 \\
Prehospital time & -0.008 & 0.882 \\
SBP & -0.051 & 0.325 \\
DBP & -0.027 & 0.607 \\
Heart rate & 0.163 & 0.002 \\
Temperature & -0.002 & 0.970 \\
Respiratory rate & 0.062 & 0.232 \\
GCS in admission & -0.204 & $<0.001$ \\
Shock & 0.204 & $<0.001$ \\
WBC & 0.237 & $<0.001$ \\
Platelet & -0.264 & $<0.001$ \\
Hemoglobin & -0.030 & 0.562 \\
Albumin & -0.126 & 0.015 \\
Glucose & 0.285 & $<0.001$ \\
Chlorine & 0.286 & $<0.001$ \\
Cholesterol & -0.184 & $<0.001$ \\
Total bilirubin & 0.037 & 0.478 \\
Serum urea & 0.291 & $<0.001$ \\
Serum creatinine & 0.523 & $<0.001$ \\
Hypertonic saline & -0.158 & 0.002 \\
Mannitol & -0.010 & 0.846 \\
Glycerol fructose & -0.037 & 0.480 \\
Furosemide & 0.002 & 0.965 \\
\hline SBP systic & \\
\hline & &
\end{tabular}

SBP: systolic blood pressure; DBP: diastolic blood pressure; GCS: Glasgow Coma Scale; WBC: white blood cell.

volemia or the surge of catecholamine after brain injury. Most of significant laboratory parameters in baseline comparison showed no statistical significance in multivariate logistic regression analysis. Only serum creatinine and SUA were identified as risk factors for AKI. Moreover, bivariate correlation analysis showed that SUA was moderately correlated with serum creatinine. Therefore, we, respectively, draw the ROC curve of SUA and serum creatinine and found that there was no statistical difference in their AUC value. The single serum creatinine level was previously considered an essential element for diagnosing AKI. However, a single assessment of serum creatinine as reflection of renal filtration function is applicable for patients with a steady state but not for those critically ill patients. Influenced by fluid dilution and blood loss which commonly occur in critically ill patients, the single serum creatinine could not sensitively and promptly reflect the acute fluctuation of estimated glomerular filtration rate (eGFR). Recently, an increasing number of studies suggested that the change in renal function from baseline was better in assessing renal injury than single creatinine cutoffs $[6,23]$. In our study, we found that combining SUA and serum creatinine could improve the accuracy of identifying patients with high risk of developing AKI after TBI. Considering drugs for reducing ICP, our study 
TABLE 4: Comparisons of the AUC value of different factors and models to predict AKI in the training cohort and validation cohort.

\begin{tabular}{|c|c|c|c|c|c|}
\hline & AUC & Sensitivity & Specificity & Standard deviation & $95 \% \mathrm{CI}$ \\
\hline \multicolumn{6}{|l|}{ Training set } \\
\hline Uric acid & 0.850 & 0.899 & 0.681 & 0.023 & $0.805-0.895$ \\
\hline Serum creatinine & 0.881 & 0.734 & 0.919 & 0.025 & $0.833-0.929$ \\
\hline Uric acid+creatinine & 0.917 & 0.823 & 0.871 & 0.019 & $0.880-0.954$ \\
\hline Model 1 & 0.935 & 0.899 & 0.833 & 0.016 & $0.903-0.967$ \\
\hline GCS & 0.686 & 0.376 & 0.924 & 0.030 & $0.627-0.745$ \\
\hline \multicolumn{6}{|l|}{ Validation set } \\
\hline Uric acid & 0.869 & 0.909 & 0.771 & 0.035 & $0.801-0.938$ \\
\hline Serum creatinine & 0.868 & 0.773 & 0.880 & 0.042 & $0.785-0.951$ \\
\hline Uric acid+creatinine & 0.905 & 0.773 & 0.904 & 0.035 & $0.836-0.974$ \\
\hline Model 1 & 0.900 & 0.773 & 0.880 & 0.036 & $0.829-0.971$ \\
\hline GCS & 0.610 & 0.783 & 0.476 & 0.069 & $0.475-0.745$ \\
\hline
\end{tabular}

GCS: Glasgow Coma Scale; AUC: area under the ROC curve; CI: confidence interval. Model 1 is composed of heart rate, shock, serum creatinine, and uric acid.

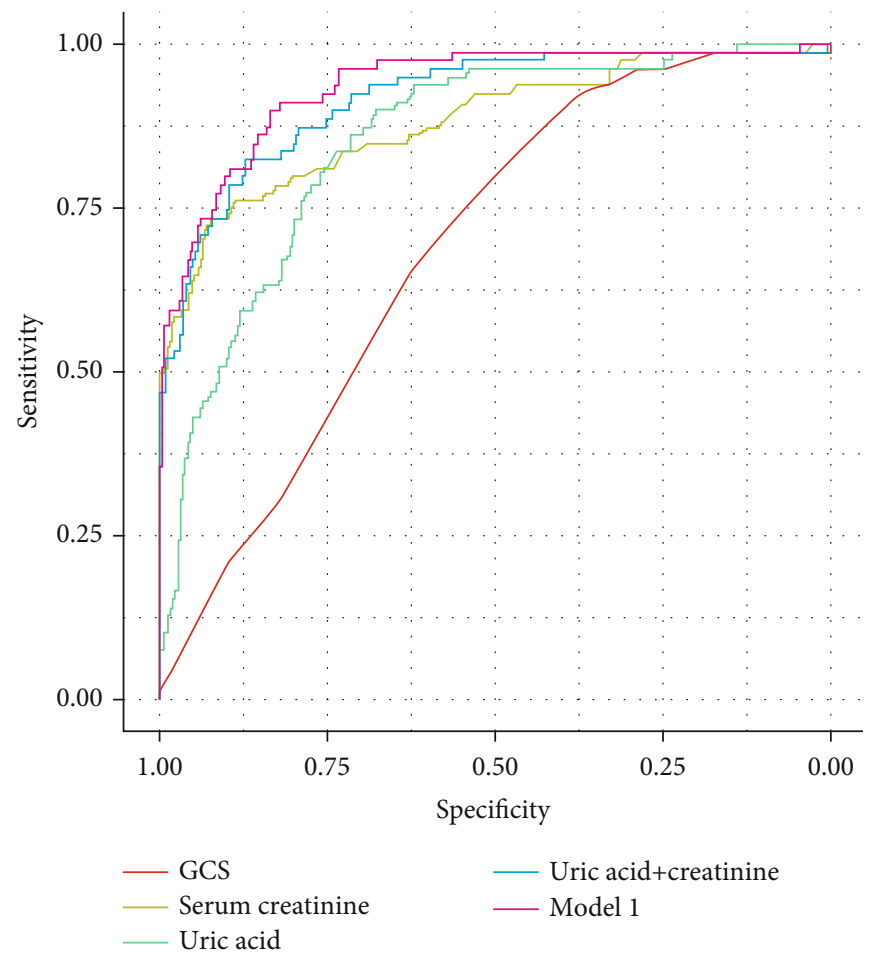

FIGURE 1: ROC curves of risk factors and constructed model 1 in the training cohort.

showed that the utilization rate of hypertonic saline was higher in the non-AKI group. Suitable use of hypertonic saline was beneficial for maintaining hemodynamic stability and renal perfusion pressure, which in turn decrease the probabilities of developing AKI. The percentage of patients using furosemide was higher in the AKI group, though without significance. This fact emphasized that unreasonable use of furosemide could lead to AKI due to hypovolemia. It has been demonstrated that accumulative doses of furosemide and mannitol were independent risk factors for AKI after cerebral trauma [24]. Nevertheless, our results did not show that the use of mannitol was associated with increased prob- abilities of AKI. This may be explained by the defect that chronological sequence of using mannitol and occurrence of AKI was not recorded. Finally, the fact that in-hospital mortality was obviously higher in the AKI group was consistent with previous studies. One cause of increased mortality in the AKI group was that renal dysfunction could aggravate cerebral edema [25]. In addition, patients who developed AKI had lower GCS which usually indicated higher severity of TBI. The 90-day GOS of the AKI group was undoubtedly lower than that of the non-AKI group. A finding that contradicted with previous studies was that AKI would shorten the length of hospital stay. A reasonable explanation was that the 


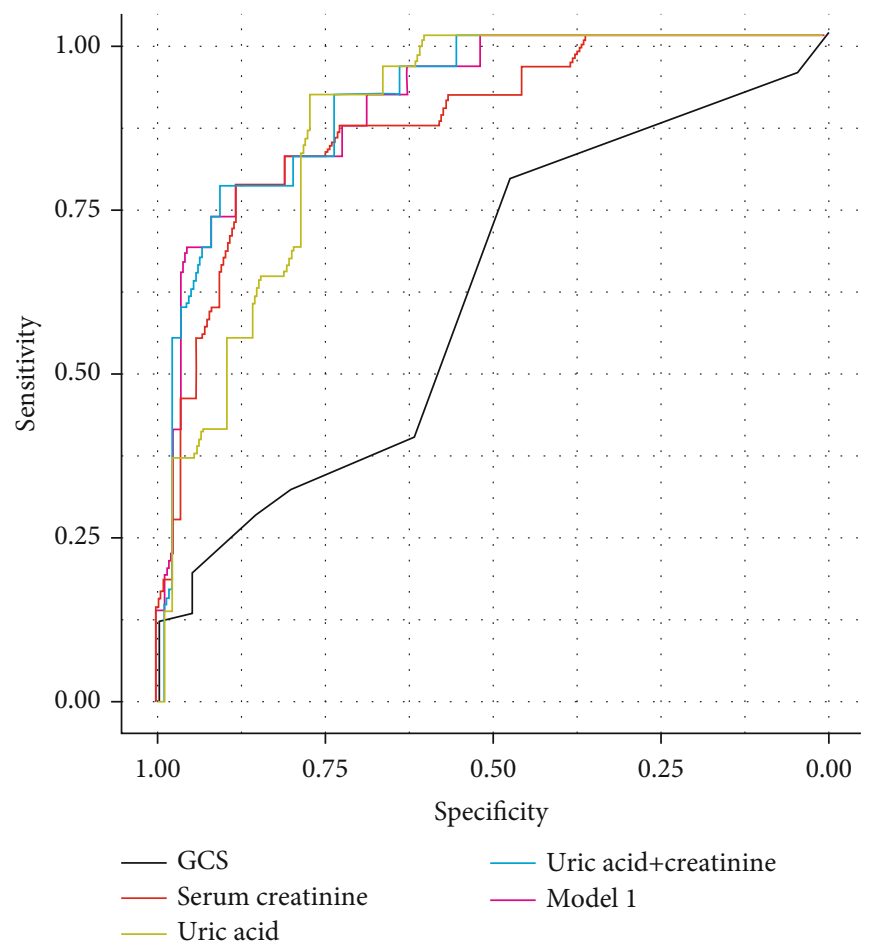

FIGURE 2: ROC curves of risk factors and constructed model 1 in the validation cohort.

mortality of the AKI group in our study was too high to significantly shorten survival time.

The mechanism of systemic complications after TBI is diversified including direct effects of brain injury as well as side effects of therapy which could initiate or exacerbate systemic complications [26]. Massive release of catecholamine and neuroinflammation caused by brain injury may lead to systemic complications including AKI [4]. Furthermore, various therapy strategies targeted on maintaining and improving cerebral physiological parameters may concomitantly impair renal function.

In humans, uric acid is an end product of catabolism of purine nucleotides arising from endogenous and exogenous sources [27]. TBI triggers catabolic processes of a series of substances including adenosine triphosphate (ATP), a major part of endogenous purine nucleotides. Studies have illustrated that accumulating uric acid impaired renal function not only by intratubular crystal precipitation but also through inducing oxidative stress and renal inflammation [14]. Uric acid could activate the renin-angiotensin system, reduce nitric oxide (NO) release, and inhibit NO synthase 1. As a result, renal ischemia and hypertension secondary to renal vasoconstriction may accelerate the development of AKI [28]. Furthermore, through inducing the expression of C-reactive protein (CRP) and monocyte chemoattractant protein-1 (MCP-1) $[29,30]$ and stimulating the proliferation of vascular smooth muscle cells, uric acid plays a proinflammatory role in impairing autoregulation of renal blood flow and reducing the GFR $[31,32]$.

The predictive and therapeutic role of uric acid has been widely studied in brain injury patients including ischemic stroke, intracerebral hemorrhage, and TBI $[12,33,34]$. One study found that low SUA level within four hours after initial injury was associated with increased mortality in TBI patients [33]. Another study made a conclusion that low SUA level which indicated more consumption by injured brain tissue was beneficial for a favorable outcome in TBI patients [11]. And exogenous supplement of uric acid could improve sensorimotor functional recovery, spatial learning, and memory in CCI mice. However, the time range of SUA assessment in the latter study was extended to three days after initial injury. We speculated that the different time points of SUA assessment lead to the opposite conclusion in these two studies. Early low SUA level indicated no enough production of neuroprotective uric acid and therefore led to a poor outcome in TBI patients. But subsequent non-earlystage low SUA could indicate more consumption of uric acid which was associated with a favorable outcome in TBI patients. However, these two studies did not evaluate the effect of endogenous SUA level and exogenous uric acid administration on AKI following TBI. Previous studies have verified the association between SUA and the occurrence of AKI in various patients including those undergoing radical cystectomy and those undergoing cardiac surgery [15-17]. Our study demonstrated that high SUA was a potent risk factor for AKI following TBI. In addition, it is generally established that AKI is an independent risk factor for mortality in patients with TBI. Previous clinical trials have verified the beneficial effect of endogenous infusion of uric acid on preventing early poor progression and alleviating oxidative stress in ischemic stroke patients [35-37]. However, the SUA level after infusion of uric acid for neuroprotection was not investigated, and the correlation between SUA level and the occurrence of AKI was not evaluated in these trials. 
Consequently, it is worthwhile to evaluate whether the neuroprotective effect of uric acid supplement on the brain is greater than the adverse effect of developing AKI and discover a rational dose to avoid renal dysfunction in future study. This contradiction perfectly illustrates the principle that treatment aimed at brain injury should focus not only on the brain but also on other organs including the kidney.

\section{Limitations}

Firstly, because this was a single-center observational study, selection bias was unavoidable. Secondly, we did not collect the history of underlying diseases and record of using nephrotoxic drugs which may influence renal function and uric acid metabolism. Thirdly, AKI was not staged so that we could not discover the relationship between the SUA level and AKI stage. Finally, the time of the occurrence of AKI was not recorded so that we could not exactly evaluate the casual relationship between AKI and usage of drugs for reducing ICP.

\section{Conclusions}

SUA is an effective marker in predicting AKI following TBI. Combining SUA and serum creatinine is beneficial for physicians to evaluate renal function in TBI patients.

\section{Data Availability}

The data used and analyzed in this study are available from the corresponding author on reasonable request.

\section{Conflicts of Interest}

All authors certify that they have no financial or nonfinancial interest in the subject matter or materials discussed in this manuscript.

\section{Authors' Contributions}

Ruo Ran Wang and Min He contributed equally to this study.

\section{Acknowledgments}

We would like to express our gratitude to Xiaoqi Xie and Xiaofeng $\mathrm{Ou}$ for their work in collecting the clinical data.

\section{Supplementary Materials}

The baseline characteristics of the training cohort and validation cohort. (Supplementary materials)

\section{References}

[1] A. A. Hyder, C. A. Wunderlich, P. Puvanachandra, G. Gururaj, and O. C. Kobusingye, "The impact of traumatic brain injuries: a global perspective," NeuroRehabilitation, vol. 22, no. 5, pp. 341-353, 2007.

[2] The Lancet Neurology, "Traumatic brain injury: time to end the silence," The Lancet Neurology, vol. 9, no. 4, p. 331, 2010.
[3] D. A. Zygun, J. B. Kortbeek, G. H. Fick, K. B. Laupland, and C. J. Doig, "Non-neurologic organ dysfunction in severe traumatic brain injury," Critical Care Medicine, vol. 33, no. 3, pp. 654-660, 2005.

[4] H. B. Lim and M. Smith, "Systemic complications after head injury: a clinical review," Anaesthesia, vol. 62, no. 5, pp. 474482, 2007.

[5] K. Goyal, A. Hazarika, A. Khandelwal et al., "Non-neurological complications after traumatic brain injury: a prospective observational study," Indian Journal of Critical Care Medicine, vol. 22, no. 9, pp. 632-638, 2018.

[6] E. M. Moore, R. Bellomo, A. Nichol, N. Harley, C. Macisaac, and D. J. Cooper, "The incidence of acute kidney injury in patients with traumatic brain injury," Renal Failure, vol. 32, no. 9, pp. 1060-1065, 2010.

[7] N. Li, W. G. Zhao, and W. F. Zhang, "Acute kidney injury in patients with severe traumatic brain injury: implementation of the acute kidney injury network stage system," Neurocritical Care, vol. 14, no. 3, pp. 377-381, 2011.

[8] N. Li, W. G. Zhao, F. L. Xu, W. F. Zhang, and W. T. Gu, "Neutrophil gelatinase-associated lipocalin as an early marker of acute kidney injury in patients with traumatic brain injury," Journal of Nephrology, vol. 26, no. 6, pp. 1083-1088, 2013.

[9] L. Corral, C. F. Javierre, J. L. Ventura, P. Marcos, J. I. Herrero, and R. Manez, "Impact of non-neurological complications in severe traumatic brain injury outcome," Critical care, vol. 16, no. 2, p. R44, 2012.

[10] M. Ahmed, K. Sriganesh, B. Vinay, and G. S. Umamaheswara Rao, "Acute kidney injury in survivors of surgery for severe traumatic brain injury: incidence, risk factors, and outcome from a tertiary neuroscience center in India," British Journal of Neurosurgery, vol. 29, no. 4, pp. 544-548, 2015.

[11] H. Liu, J. He, J. Zhong et al., "Clinical and basic evaluation of the prognostic value of uric acid in traumatic brain injury," International Journal of Medical Sciences, vol. 15, no. 10, pp. 1072-1082, 2018.

[12] Z. Wang, Y. Lin, Y. Liu et al., "Serum uric acid levels and outcomes after acute ischemic stroke," Molecular Neurobiology, vol. 53, no. 3, pp. 1753-1759, 2016.

[13] M. Kuwabara, P. Bjornstad, I. Hisatome et al., "Elevated serum uric acid level predicts rapid decline in kidney function," American Journal of Nephrology, vol. 45, no. 4, pp. 330-337, 2017.

[14] M. Shimada, R. J. Johnson, W. S. May et al., "A novel role for uric acid in acute kidney injury associated with tumour lysis syndrome," Nephrology Dialysis Transplantation, vol. 24, no. 10, pp. 2960-2964, 2009.

[15] T. Kaufeld, K. A. Foerster, T. Schilling et al., "Preoperative serum uric acid predicts incident acute kidney injury following cardiac surgery," BMC Nephrology, vol. 19, no. 1, p. 161, 2018.

[16] K. W. Joung, S. S. Choi, Y. G. Kong et al., "Incidence and risk factors of acute kidney injury after radical cystectomy: importance of preoperative serum uric acid level," International Journal of Medical Sciences, vol. 12, no. 7, pp. 599-604, 2015.

[17] A. A. Ejaz, T. M. Beaver, M. Shimada et al., "Uric acid: a novel risk factor for acute kidney injury in high-risk cardiac surgery patients?," American Journal of Nephrology, vol. 30, no. 5, pp. 425-429, 2009.

[18] K. Uhlig, J. Calvo-Broce, A. Deo, and A. Earley, "Section 2: AKI definition," Kidney International Supplements, vol. 2, no. 1, pp. 19-36, 2012. 
[19] L. Mascia, Y. Sakr, D. Pasero et al., "Extracranial complications in patients with acute brain injury: a post-hoc analysis of the SOAP study," Intensive Care Medicine, vol. 34, no. 4, pp. 720-727, 2008.

[20] R. Lefering, T. Paffrath, R. Linker, B. Bouillon, and E. A. M. Neugebauer, "Head injury and outcome-what influence do concomitant injuries have?," The Journal of Trauma, vol. 65, no. 5, pp. 1036-1044, 2008.

[21] M. B. Skrifvars, E. Moore, J. Mårtensson et al., "Erythropoietin in traumatic brain injury associated acute kidney injury: a randomized controlled trial," Acta Anaesthesiologica Scandinavica, vol. 63, no. 2, pp. 200-207, 2019.

[22] J. H. Siegel, "The effect of associated injuries, blood loss, and oxygen debt on death and disability in blunt traumatic brain injury: the need for early physiologic predictors of severity," Journal of Neurotrauma, vol. 12, no. 4, pp. 579590, 1995.

[23] Z. Ricci, D. Cruz, and C. Ronco, "The RIFLE criteria and mortality in acute kidney injury: a systematic review," Kidney International, vol. 73, no. 5, pp. 538-546, 2008.

[24] L. Fang, H. You, B. Chen et al., "Mannitol is an independent risk factor of acute kidney injury after cerebral trauma: a case-control study," Renal Failure, vol. 32, no. 6, pp. 673679, 2010.

[25] A. Davenport, "Renal replacement therapy in the patient with acute brain injury," American Journal of Kidney Diseases: The Official Journal of the National Kidney Foundation, vol. 37, no. 3, pp. 457-466, 2001.

[26] D. Zygun, "Non-neurological organ dysfunction in neurocritical care: impact on outcome and etiological considerations," Current Opinion in Critical Care, vol. 11, no. 2, pp. 139-143, 2005.

[27] G. Ndrepepa, "Uric acid and cardiovascular disease," Clinica chimica acta; international journal of clinical chemistry, vol. 484, pp. 150-163, 2018

[28] A. Ejaz, W. Mu, D.-H. Kang et al., "Could Uric Acid Have a Role in Acute Renal Failure?," Clinical Journal of the American Society of Nephrology, vol. 2, no. 1, pp. 16-21, 2006.

[29] D. H. Kang, S. K. Park, I. K. Lee, and R. J. Johnson, "Uric acidinduced C-reactive protein expression: implication on cell proliferation and nitric oxide production of human vascular cells," Journal of the American Society of Nephrology: JASN, vol. 16, no. 12, pp. 3553-3562, 2005.

[30] T. Umekawa, N. Chegini, and S. R. Khan, "Increased expression of monocyte chemoattractant protein-1 (MCP-1) by renal epithelial cells in culture on exposure to calcium oxalate, phosphate and uric acid crystals," Nephrology, dialysis, transplantation: official publication of the European Dialysis and Transplant Association-European Renal Association, vol. 18, no. 4, pp. 664-669, 2003.

[31] P. Devarajan, "Update on mechanisms of ischemic acute kidney injury," Journal of the American Society of Nephrology: JASN, vol. 17, no. 6, pp. 1503-1520, 2006.

[32] L. G. Sanchez-Lozada, E. Tapia, J. Santamaria et al., "Mild hyperuricemia induces vasoconstriction and maintains glomerular hypertension in normal and remnant kidney rats," Kidney International, vol. 67, no. 1, pp. 237-247, 2005.

[33] M. Hatefi, "Association of serum uric acid level with the severity of brain injury and patient's outcome in severe traumatic brain injury," Journal of Clinical and Diagnostic Research, vol. 10, no. 12, pp. 20-24, 2016.
[34] A. Karagiannis, D. P. Mikhailidis, K. Tziomalos et al., "Serum uric acid as an independent predictor of early death after acute stroke," Circulation Journal: Official Journal of the Japanese Circulation Society, vol. 71, no. 7, pp. 1120-1127, 2007.

[35] S. Amaro, D. Cánovas, M. Castellanos et al., "The URICOICTUS study, a phase 3 study of combined treatment with uric acid and rtPA administered intravenously in acute ischaemic stroke patients within the first $4.5 \mathrm{~h}$ of onset of symptoms," International Journal of Stroke, vol. 5, no. 4, pp. 325-328, 2010.

[36] K. Kikuchi, K. Setoyama, E. Tanaka et al., "Uric acid enhances alteplase-mediated thrombolysis as an antioxidant," Scientific Reports, vol. 8, no. 1, p. 15844, 2018.

[37] S. Amaro, C. Laredo, A. Renú et al., "Uric acid therapy prevents early ischemic stroke progression: a tertiary analysis of the URICO-ICTUS trial (efficacy study of combined treatment with uric acid and r-tPA in acute ischemic stroke)," Stroke, vol. 47, no. 11, pp. 2874-2876, 2016. 